\title{
Case Study: Not a Normal Hair Case- an Alpaca Hair Comparison
}

\author{
Emily Weber ${ }^{1}$
}

1. Hamilton County Coroner's Office Crime Laboratory - Trace Evidence Section, 3159 Eden Ave., Cincinnati, Ohio

For a number of months, a woman's farm animals had been found deceased. One morning she found her alpaca lifeless. Her first suspicion was her estranged husband, whom she filed a restraining order against. She contacted the local Sheriff's Office to report the crime.

The husband's work boots were confiscated by officers. They wanted to determine if alpaca hair was present on the boots to place the husband at the farm, which the restraining order prohibited. In addition, known hair samples were collected from the deceased alpaca.

Field research was conducted in preparation for this case. A trip to the East Fork Alpaca Farm was warranted in order to collect known samples from the two types of alpacas: Huacaya and Suri. Characteristics of the Huacaya alpaca fleece include a crimpy appearance as it grows perpendicularly from the alpaca's body, giving the hair a "poofy" or fluffed apearance [1]. Suri alpaca fleece, on the other hand, has characteristically long, lustrous locks or ringlets which move freely as the animal walks [2]. Alpaca hair was collected from multiple locations on the two types of animals including the back, belly, leg, neck, and wool cap, to account for any microscopic variation.

Alpaca hair typically consists of two different types of hair: guard hairs and fleece hair. Guard hairs are stiff, straight, and highly medullated [3]. These medullated guard hairs are not desirable in finished products [4]. Fleece hairs are fine and light with little to no medullation, making them the desirable portion of the alpaca's coat.

Known Huacaya and Suri hair "standards" were mounted on microscope slides and examined under a Leica DM2500P comparison microscope. The microscopic characteristics of these known alpaca hairs were observed and noted. The hairs from the deceased alpaca, as well as the hairs collected from the suspect's boots were mounted on microscope slides and compared to the hair "standards".

Examination of thirty-one hairs from the soles of the work boots were determined to be animal hairs (Figure $4 \& 5$ ). However, the medullas of these animal hairs were different than the medullas observed in the known alpaca hair "standards" (Figure $7 \& 8$ ). Thick, vacuolated medullas were noted in the animal hairs from the work boots, while the medullas of the known alpaca standards appeared more human-like with amorphous medullas [5]. The hair from the deceased alpaca also exhibited this amorphous medulla (Figure 6). Due to the microscopic differences observed in the animal hairs from the boots, a correspondence to the deceased alpaca could not be made.

\section{References:}

[1] Bat, Linda and Rus Hinman. "How to Evaluate Alpaca Fiber." Delphi Alpacas, 2008. Web. 15 Dec. 2015. 
[2] Ziek, Barbara. "What's So Special About Alpaca Fiber?" Wild Hair Alpacas LLC, 2015. Web. 14 Dec. 2015.

[3] Bolewicz, Barry and Linda Bolewicz. "Basics about Alpaca Fleece.” EasyGo Farm. Web. 14 Dec. 2015.

[4] Holt, Cameron. "Fibre Testing for Alpaca Breeders." C Holt, 2007. Web. 15 Dec. 2015.

[5] Deedrick, Douglas, and Sandra Koch. "Microscopy of Hair Part II: A Practical Guide and Manual for Animal Hairs." Forensic Science Communications 6.3 (2004).

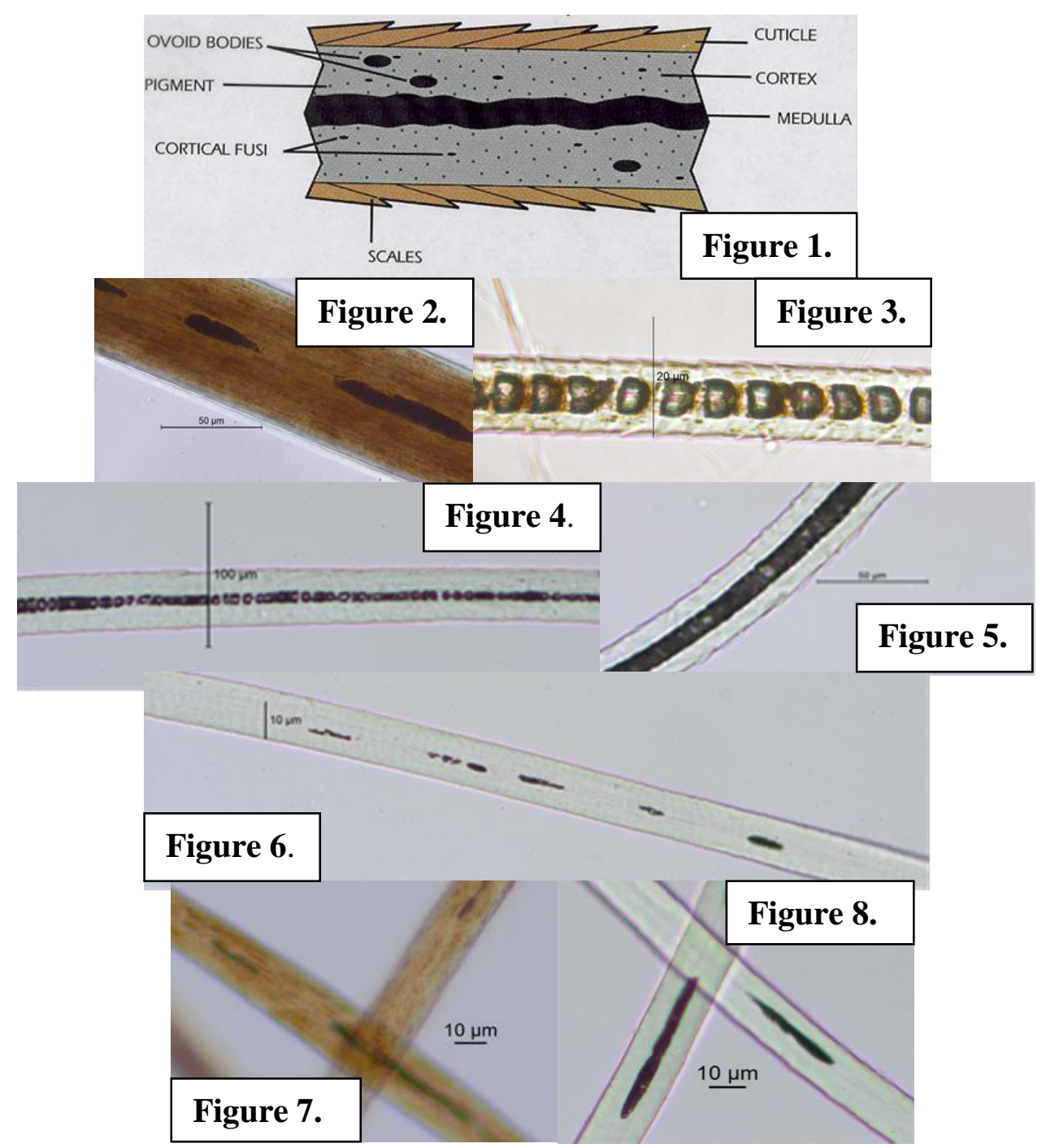

Figure 1. Schematic diagram of a human hair

Figure 2. Human hair

Figure 3. Animal hair

Figure 4. Unknown hair from boot, exhibiting a uniserial-ladder medulla

Figure 5. Unknown hair from boot, exhibiting a thick, vacuolated medulla

Figure 6. Known hair from the deceased alpaca, exhibiting an amorphous medulla

Figure 7. Known hair from a Suri alpaca, exhibiting an amorphous medulla

Figure 8. Known hair from a Huacaya alpaca, exhibiting an amorphous medulla 